10.2478/v10367-012-0005-9

ALAN GRANT, GEORGE SHAW, NICK WARD

The General Lighthouse Authorities of the United Kingdom and Ireland

\title{
THE EFFECT OF SPACE WEATHER ON MARITIME AIDS-TO-NAVIGATION SERVICE PROVISION
}

\begin{abstract}
The General Lighthouse Authorities of the United Kingdom and Ireland (GLA) provide marine Aids-to-Navigation (AtoNs) for the benefit and safety of all mariners within their waters. These AtoNs include traditional lighthouses, buoys and various radionavigation systems.

It is recognised that GPS, or more generally Global Navigation Satellite Systems (GNSS), have become the primary means of obtaining Position, Navigation and Timing (PNT) information at sea. Mariners may have come to believe that GPS is infallible, yet it is known to be vulnerable to solar activity, interference and system failures.

The Sun continuously releases random bursts of energy and highly charged particles. The impact of these emissions on the Earth is known as space weather. Bursts of electromagnetic energy can result in radio blackouts; bursts of high energy particles can increase ionising radiation and affect space craft performance; and bursts of magnetised plasma can result in the degradation and potential loss of radionavigation signals on Earth.

The amount of solar activity is linked with the natural sunspot cycle, which shows the number of sunspots peak approximately every 11 years, with the next peak due in 2013. Sunspots occur almost continuously, but normally give rise to weak solar events that generally go by unnoticed. However significant storms can occur at any time and as we approach peak activity, the number of space weather events will increase.

AtoNs generally report their position using radio systems, whether through the Automatic Identification System (AIS), public networks or dedicated links; and often use GNSS to calculate their position and to obtain timing information. Mariners use GNSS for PNT information; with this information integrated into many different bridge systems. Powerful solar storms can affect GNSS performance and the reception of their signals, which may lead to different correlated events, both on and off the ship. During very extreme and very rare storms, national power systems may be switched off to protect the infrastructure which would clearly affect those services relying on mains power without backup.

This paper reports the output of a study into the potential effects of space weather on GLA AtoN service provision and presents mitigating actions, where appropriate. The outcome of this study will help the GLA to continue to provide the highest level of AtoN availability, helping to ensure the safety of all mariners.
\end{abstract}

\section{Keywords:}

marine aids to navigation, space weather, solar activity. 


\section{INTRODUCTION}

The General Lighthouse Authorities of the United Kingdom and Ireland (GLAs) comprise Trinity House, The Commissioners of Irish Lights and The Northern Lighthouse Board. Between them, they have the statutory responsibility to provide marine Aids-to-Navigation (AtoNs) around the coast of England and Wales, all of Ireland and Scotland, respectively. AtoNs take many forms, from the more traditional lighthouse to radio navigation systems, including the use of new GNSS when they become available.

It is well known that space weather can affect GNSS performance, radio systems and power networks on Earth and therefore it is incumbent on the GLAs, as safety of life service providers to understand the potential impact of space weather on our AtoNs.

According to the US National Space Weather Program, the term space weather refers to the conditions on the sun and in the solar wind, magnetosphere, ionosphere, and thermosphere that can influence the performance and reliability of space-borne and ground-based technological systems and can endanger human life or health [17].

Space weather events occur due to disturbances within the sun's magnetic field which result in sunspots. Sunspots are the visualisation of these disturbances, as they reach the surface of the sun and while sunspots themselves don't necessarily result in any of these events, they are an indication that such an event may occur.

\section{SOLAR CYCLE}

The number of sunspots observed each year follows an approximate 11 year cycle, known as the solar cycle. The last peak occurred in 2000 and the next peak is slightly delayed and expected to peak mid 2013, according to the latest prediction (fig. 1). From the predicted values in this figure, it is reasonable to expect that the next peak will see fewer sunspots than that experienced in 2000 and therefore it could be misconstrued that impact will be less. This is not necessarily the case.

The severity of space weather events is stochastic in nature meaning that extreme storms can occur at any time. Therefore even if the peak in sunspot numbers observed in 2013 is lower than the last peak, it could be that the storm levels are more severe; alternatively they may be weaker, no one knows what will actually happen. 
Looking back at the last peak in 2000, there was a major solar radiation storm in July 2000, the Bastille Day storm, which resulted in a significant geomagnetic storm. Later in 2003, the so called Halloween storms occurred during the waning phase of the sunspot cycle and included the largest x-ray flare since 1976. Therefore, while the peak in the solar cycle indicates more solar activity, significant events can occur at any time.

\section{SPACE WEATHER EVENTS}

Space weather events can be generally categorised into three main types, where each describes a different phenomena, but can result in similar outcomes. These types are:

— radio blackouts caused by solar flares;

— seomagnetic storms;

- Solar Energetic Particles (SEP) or Solar radiation storms.

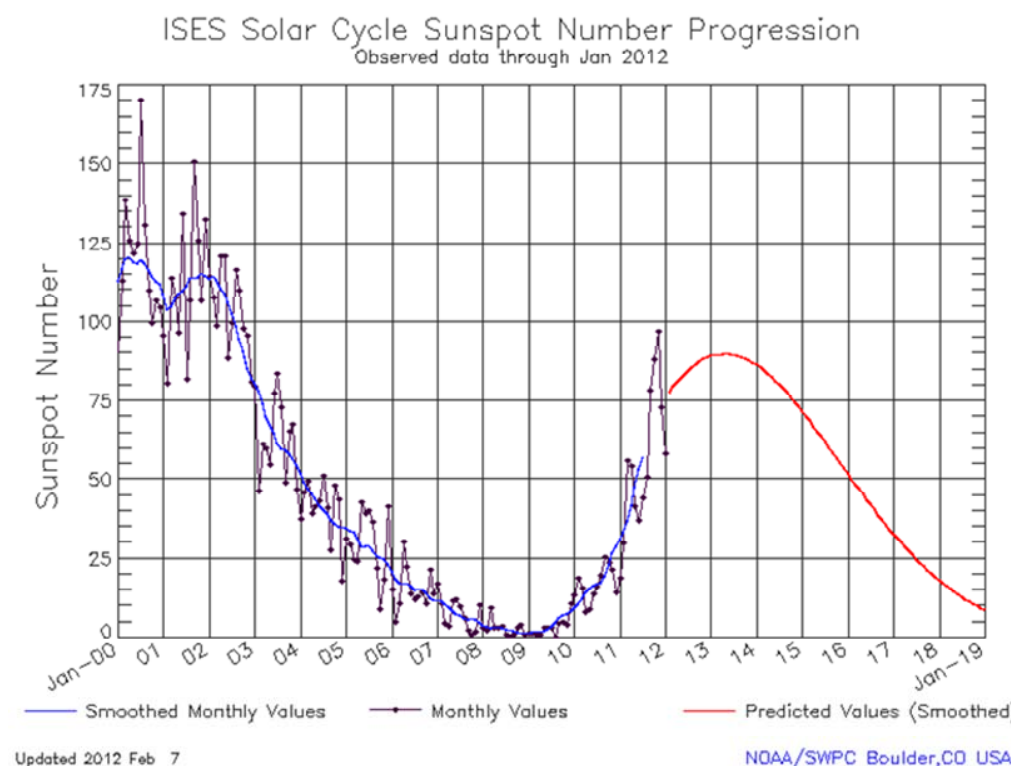

Fig. 1. NOAA Solar cycle showing the recorded sunspots and the predicted values [9]

The GLAs are not experts in space weather, but by reviewing the available literature $[1,5,7-11,14-16]$, each event is briefly described in turn. 


\section{Solar Flares \& Radioblackouts}

Solar flares are eruptions on the Sun surface where disturbances in the magnetic field cause the release of radio emissions. They can exist for several tens of second through to a few hours and release X-ray, ultraviolet and other radio emissions across all frequencies of the electromagnetic spectrum. They can produce ionospheric disturbances for the sunlit part of the Earth by producing sudden increases in the total electron content (TEC) and although these occurrences are often short lived they can cause additional tracking problems for GNSS receivers. Very strong flares can produce solar radio bursts or blackouts which may affect the reception of high frequency signals (3-30 MHz), and VHF (30-300 MHz), however at the latter frequencies it is reported to mainly affect operational range and not prevent signal reception within range.

\section{Geomagnetic storms}

Geomagnetic storms are strong disturbances to the Earth's magnetic field caused by magnetically polarised energy from the Sun. Such energy can be released by Coronal Mass Ejections (CMEs) which rapidly shower the Earth with charged particles one to four days after the event. As the emitted plasma cloud reaches Earth, the magnetosphere attempts to divert this energy towards the poles, but the polarisation of the incoming energy determines its success.

Geomagnetic storms can be visible to the human eye. The aurora observed at the poles is produced by the interaction of the Earth's magnetosphere and the charged particles from space. During large storms, the amount of charged particles increases and the aurora is visible at lowering latitudes. Figure 2 provides an estimate as to what level storm (graded from $\mathrm{Kp}=5$ Minor to $\mathrm{Kp}=9$ Extreme) causes the aurora to be seen at which latitude across Europe. This mapping gives an indication as to where the impact of geomagnetic events could be observed.

During these storms very large electrical currents of up to a million amperes can flow through the ionosphere and magnetosphere, which can change the direction of the Earth's magnetic field at the surface by up to 1 or 2 degrees, mainly in the aurora regions, although these effects can extend to mid-latitudes. The changes to the magnetic field can disrupt radio communications and induce electrical currents in long cables, through a process known as Geomagnetic Induced Currents (GIC). High levels of GICs in long cables, such as those used in power distribution networks, can damage equipment and lead to power failure. To prevent damage, power companies isolate their equipment in response to warnings provided by space weather monitoring services. 


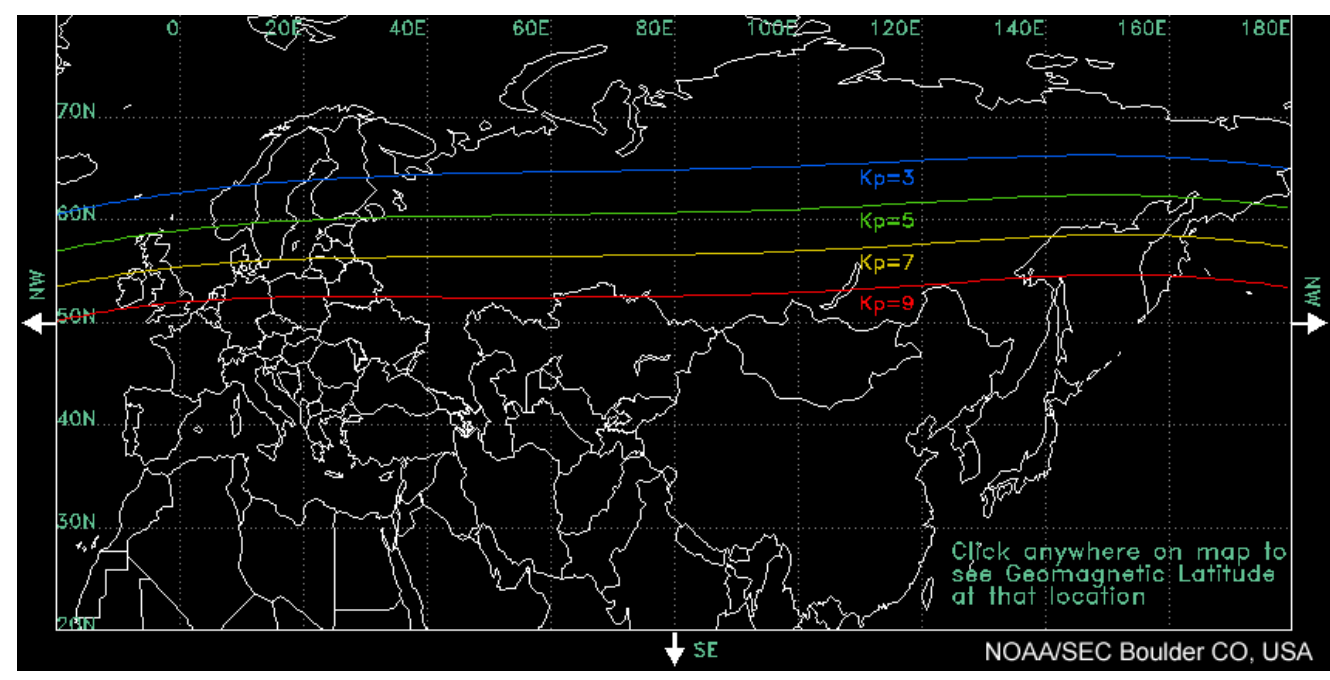

Fig. 2. A map of Europe showing the estimated edge of the aurora under different geomagnetic storm conditions [18]

\section{Solar Energetic Particles (solar radiation storms)}

The Sun occasionally emits large quantities of energetic protons and electrons. These energetic particle events can last for a few days at a time and they can affect both ground-based and space-based systems. These particles can cause single event upsets (SEU) in satellite and ground based electronics. These cause data stored in memory to change state at the bit level, corrupting data and affecting the operation of satellites or systems.

\section{Ionospheric Response}

Solar flares and CMEs can increase the number of free electrons within the ionosphere (known as ionospheric storms), which in turn effects the refraction and diffraction of the GNSS signal as it propagates through the ionosphere. Unlike the impact of other space weather events, ionospheric storms may affect the whole of the Earth and not just the sunlight side.

Irregularities in TEC may produce scintillations, where the TEC changes by varying amounts, depending on latitude. Seemingly random areas of the upper ionosphere can have different TEC densities and can cause radio waves to be misdirected. This can lead to both amplitude and phase variations in the GNSS signals and ultimately prevent signals from being tracked. Large increases and decreases in the TEC directly influence the accuracy of single-frequency GNSS receivers as the 
time of transit for GNSS data is delayed, which in turn relates to poorer accuracy. Dual-frequency GNSS receivers measure the effect of the ionosphere by comparing the delay of data on two frequencies and can mitigate the effects to a certain degree but will also be affected.

Such an example of how GNSS can be affected is given in figure 3, where the carrier-to-noise ratio $\left(\mathrm{C} / \mathrm{N}_{\mathrm{o}}\right)$ for two GPS satellites was tracked for a short period. One is suffering the effect of scintillation (PRN7) while the other does not and gives a constant figure (PRN8). Specialist equipment was used to record this data and it was reported that most GPS receivers would not be able to track satellites when the C/No ratio drops below 27-28 dB.

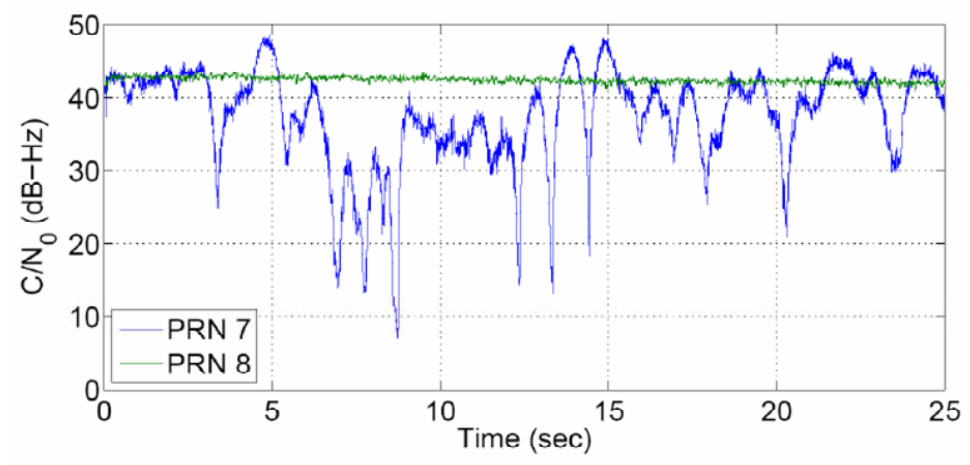

Fig. 3. The carrier-to-noise ratios of two GPS satellites during a period of scintillation [7]

Ionospheric scintillation is generally limited to three bands of latitude:

- equatorial $\left( \pm 10^{\circ}\right.$ geomagnetic latitude);

- aurora regions $\left( \pm 65^{\circ}-75^{\circ}\right.$ geomagnetic latitude);

- polar cap regions ( $>75^{\circ}$ geomagnetic latitude).

The GLA service area falls within the range of $45^{\circ}-65^{\circ}$ latitude (approximately $42^{\circ}-62^{\circ}$ geomagnetic latitude) and therefore the most affected areas will be to the north.

\section{REVIEW OF PREVIOUS EVENTS}

Sunspots occur almost continuously, but normally give rise to weak solar events that generally go by unnoticed. However powerful events do occur and this section provides a short overview of some events that either affected maritime services or had the potential to. 


\section{Carrington Event}

The largest space weather storm recorded, and the one that gets the most coverage, is the Carrington event in 1859. On the morning of September 1, 1859, amateur astrologer Richard Carrington observed a number of enormous dark spots on the Sun's surface that suddenly became two patches of intensely bright light. Within minutes the bright light vanished, but after a few hours their impact was felt across the globe.

Carrington had observed a massive solar flare that emitted charged particles toward Earth, resulting in a geomagnetic storm which affected the magnetosphere to make the aurora visible at lower latitudes and induced currents into the telegraph wires around the world. The power of the storm was not recorded, but it is reported to be much greater than any other storm in history [19] and is often termed a once in 300-500 year event. Such a storm today would have a significant effect on both satellite navigation and shore based operations.

\section{Quebec power outage}

In March 1989, the third strongest geomagnetic storm on record struck Earth. In less than a minute, induced currents in transmission lines caused overload safety systems to trip, closing down sections of the of the Quebec power network. Systematically different systems tripped out, to a point where the network collapsed, plunging Quebec into darkness. Electricity was unavailable for over 9 hours. Restoration was made all the more difficult by the fact that spare equipment was also affected by space weather.

\section{Halloween storms}

In 2003 during the declining phase of the solar cycle, the Sun unexpectedly burst into activity. A number of flares resulted from very large and complex sunspot groups and included one of the most intense flares ever recorded. This solar flare activity was accompanied by CMEs and strong energetic particle events (radiations storms). These resulted in extreme geomagnetic storms that caused outages in high frequency communication systems, fluctuations in power systems and minor to severe impacts on satellite systems.

To put the Halloween group of storms into context, it not only produced many major solar flares, including the largest X-ray flare recorded since 1976, but it also produced 4 different high level radiation storms and 5 geomagnetic storms of which two were considered Extreme [10]. 
The impact of these storms was felt all over the world and affected many different systems, including:

- two Inmarsat satellites required manual intervention to maintain the correct orbit while another went offline due to a CPU failure;

- some GPS users observed errors and high accuracy users, such as surveying and deep-sea drilling companies, postponed planned operations;

- the US Wide Area Augmentation System (WAAS) was significantly affected; for a 15 hour period on the $29^{\text {th }}$ October and for an 11 hour period on the $30^{\text {th }}$ October, the ionosphere was so disturbed that the vertical error limit of $50 \mathrm{~m}$ was exceeded and WAAS was unusable for precision approaches;

- power companies reported GIC across North America and Northern Europe;

— deviations of up to $7^{\circ}$ were observed on a magnetic compass in Lerwick, Scotland [12].

\section{6 radio burst}

In 2006, during a quiet point in the sunspot cycle, the Earth was exposed to the largest solar radio burst (radio blackout) ever recorded. It was the first recorded incident of a radio burst affecting GPS reception. GPS reception was affected over the sunlight side of the Earth as can be seen in figure 4.

This figure provides images detailing the performance of various GPS receivers over several minutes of $6^{\text {th }}$ December 2006. The green dots indicate receivers that were able to track 4 or more satellites and provide a position, whereas the red dots indicate those that could not track sufficient satellites. The images show the failure of receivers on the sunlight side of the Earth as the effect of the storm hit. In some instances GPS was unavailable for approximately 30 minutes.
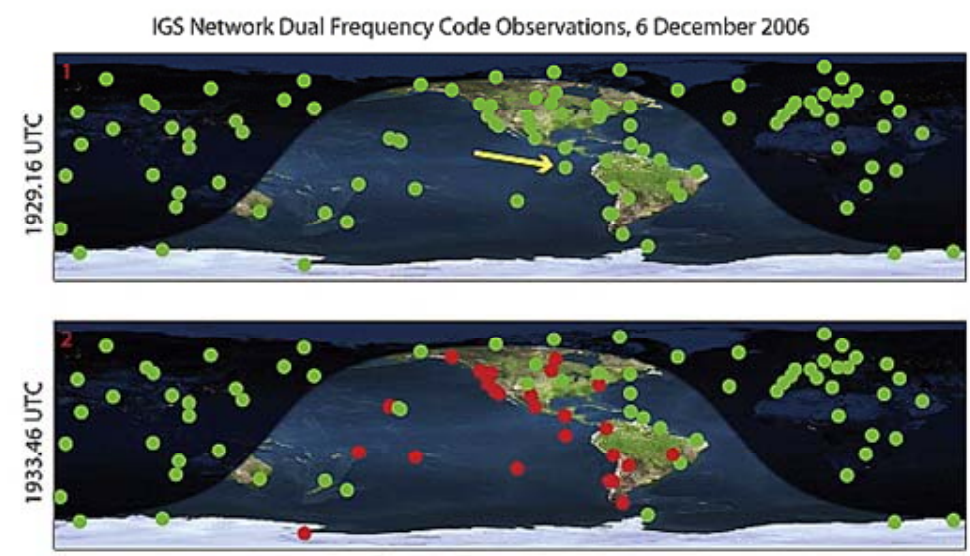

Fig. 4. Map of GPS receivers that were affected by the radio blackout. The colours indicate the stations ability to track 4 or more satellites, with red dots indicating those that lost lock [2] 


\section{LIKELIHOOD OF EVENTS}

Space weather events occur randomly, however several studies have attempted to provide a statistical overview of the probability, or likelihood, of these events occurring.

The NOAA Space Weather Scales provide an average frequency for space weather events, breaking each of the three event types into 5 categories, based on their strength. They provide the frequency of occurrence in terms of the number of storm events per solar cycle and the number of affected days. NOAA predicts that G1 'minor' geomagnetic storms will occur 1700 times during the 11 year cycle, affecting 900 days; with a G5 'extreme' geomagnetic storm occurring 4 times during the cycle, affecting 4 days.

While these figures estimate the number of events and clearly show that there will be fewer storms of the more extreme strength, they don't provide any information as to when to expect them. Due to the stochastic nature of these events it is difficult to predict when they will occur, however it is possible to follow a general trend through the numbers provided. It should be noted that these figures do not mean this number of events will happen, nor does it preclude additional events from occurring.

\section{GLA AIDS-TO-NAVIGATION}

Sufficiently strong space weather events can affect radio based navigation and communication systems, the performance of the magnetic compass and power systems; all of which have the potential to affect the operation or monitoring of GLA AtoNs.

This section reviews GLA services and considers the following in turn:

- AtoN Deployment;

- physical AtoNs;

— radionavigation systems;

- AtoN Monitoring.

\section{Aton Deployment}

The GLAs operate a fleet of buoy tenders which are used to deploy and maintain floating AtoNs. The impact of space weather on their operations can be considered typical for most mariners and impact of space weather is considered on each bridge system or operation in turn. 
GNSS based systems

The use of Position, Navigation and Timing information derived by GNSS is widespread within many vessels' navigation systems and underpins situational awareness for many mariners.

When significant storms occur, GNSS may become less precise due to greater electron content in the atmosphere which delays the propagation of signals from the satellite to the receiver. The extent of the resulting degradation in accuracy will depend on location and will be linked to the severity of the storm. Where mariners use single frequency stand alone GNSS, they may not be aware of the degradation and could continue to navigate unaware of the reduction in accuracy. It is reported that position errors up to $60 \mathrm{~m}$ could be induced by changes to the ionospheric delay [6].

Where differential services are employed, the differential process will mitigate the increase in position error, to a certain degree. When the error reaches the trigger for the reference station integrity monitor, an alarm is raised to alert the mariner of a loss of performance. Where dual frequency stand-alone GNSS receivers are used, the effect of space weather can be mitigated to some extent (as the receiver can calculate the ionospheric delay), but positional accuracy may still be affected.

Degradation and denial of GNSS could affect a large area at the same time, causing many vessels and AtoNs to lose their position, navigation and timing information. The effect on a typical vessel is expected to be similar as that experienced during the GLA GPS jamming trials [4].

\section{Dynamic positioning}

The GLA vessels use Dynamic Positioning (DP) to maintain position when deploying AtoNs, to ensure they are deployed in the correct position. DP takes several position inputs and an inertial system to hold the vessel in position by controlling the power to the engines. The impact of degraded or denied GNSS positioning is not known, however given the use of an inertial system it is expected that DP systems should be able to maintain operations during short term outages and should raise an alarm otherwise.

Further work is required to understand how the GLA vessel systems would respond to such a situation.

\section{Magnetic compass}

As noted earlier, when geomagnetic storms occur there is the potential for magnetic compasses to be affected, with deviations of up to $7^{\circ}$ observed in Lerwick, Scotland, during the Halloween storms of 2003. Clearly this could also affect a vessel's 
magnetic compass and while most vessels obtain heading information from a number of systems, including the gyro or GNSS based system, regular cross checks to the magnetic compass are taken to ensure any offset or drift error is managed.

Therefore it is important that the mariner is made aware should the magnetic compass be affected, otherwise they may erroneously interpret the difference as a greater drift and make erroneous corrections accordingly.

Voice \& data communication systems

Onboard communication systems may be affected by space weather events. High frequency (HF) and low frequency (LF) systems make use of the ionosphere for propagation and therefore can be affected by geomagnetic storms and radio blackouts. While VHF systems can also be affected, they are considered less vulnerable than the other systems [8].

Satellite communications, either voice or data, can be affected either through problems associated with the communications satellite itself (for example drag or SEUs) or through the alignment of the vessel's satellite dish to the satellite, as this is often oriented by GNSS.

\section{Physical Atons}

Physical AtoNs are those where the physical structure and/or their colours are used to convey safety information to the mariner.

\section{Buoys \& Major Floating Aids}

Buoys and Major Floating Aids (MFA) as physical AtoNs are not affected by space weather events, even during the most extreme event they will continue be available. Space weather events do however have the potential to affect the performance of any additional equipment installed on the buoy/MFA, such as an AIS AtoN and they could also affect the GLAs' ability to monitor its location and performance.

\section{Radionavigation systems}

\section{Automatic Identification System}

The Automatic Identification System (AIS) provides information from ship-to-ship and from ship-to-shore over two VHF frequencies. The GLAs make use of the AIS infrastructure to provide AtoN AIS units that enable the mariner to get access to the AtoN's location and operational information in real-time. AIS Transponders use GPS derived time to synchronise the timing of data across the network. 
Where stand-alone GPS is employed and its performance is degraded due to ionospheric effects, the AtoN's reported position may be in error. It is likely that mariners in the vicinity will also be suffering degraded GPS positions and therefore situational awareness could be affected.

Unlike their ship borne counter parts, AtoN AIS units may, or may not, synchronise to a base station in the absence of GPS derived time. Where the design means the transponder is unable to synchronise to a different time source, the unit will either transmit a known position mask to indicate loss of position, or stop transmitting altogether.

\section{Marine beacon DGPS}

The GLA marine beacon DGPS service compares the known surveyed position of the reference station with the estimated GPS position to calculate corrections for each satellite.

Where the mariner's receiver observes the same propagation delay as the reference station, the broadcast corrections will be the most effective and provide the greatest accuracy. However, as the distance between the two increases, each receiver observes its own propagation delays, due to the path from the receivers to the satellite propagating through different sections of the Earth's atmosphere and being exposed to different delays. This is known as spatial decorrelation.

DGPS corrections take into account all of the position errors associated with the transmission and propagation of the satellite signal. Therefore, if the TEC increase is fairly uniform and the DGPS baseline is not too great then the corrections will be effective. But if there are large spatial TEC gradients and the baseline is significant then the corrections may not be effective even within the limit at which that integrity alarm would be raised. Where scintillation is sufficient to prevent GNSS signal reception, the reference station and the mariner will both experience the same effect when in the same area. In the scenario where a reference station is unable to track GNSS and provide corrections, corrections from a more distant station may be available.

Where GNSS reception is denied over a large area, the DGPS service will continue to broadcast messages over the $300 \mathrm{kHz}$ radio link, albeit without correction information. This link could be used to inform the mariner of the space weather event through the use of an RTCM Type 16 message.

Medium Frequency transmissions of Marine beacon DGPS corrections are also susceptible to changes in the ionosphere, with signals propagating further at night due to refracting off the ionosphere. Space weather events may cause these effects to be observed during the day, however further work is needed to monitor the impact fully. 
e Loran

The GLAs operate a trial eLoran service that broadcasts data over a $100 \mathrm{kHz}$ radio link. This service makes use of signals refracting off the ionosphere and as such is susceptible to ionospheric effects, most notably resulting in early skywave.

Early skywave is where the eLoran skywave signal that refracts off the ionosphere arrives earlier than anticipated and affects the receiver's ability to calculate the distance to the mast. It is likely that mariners close enough to an eLoran station so that they are only using the groundwave component would still be able to use the service.

Through the use of an LF transmission, eLoran is also susceptible to radio blackouts, however it is widely anticipated that different frequencies will be affected differently and therefore any loss of service on one frequency should not be correlated with a loss on another. This was believed to be the case during the 2006 radio storm, where GPS was denied but Loran remained operational.

Further work is required to fully understand the different failure modes.

Racons

Racons (radar beacons) return a known Morse character when interrogated by a vessel's radar. While they could technically be affected by radio interference or SEUs there are no examples of space weather effecting such equipment within the literature.

It is therefore with caution that it is concluded that racons operational within GLA waters, should not be significantly affected.

\section{Lighthouses}

In a similar manner to buoy and MFA, lighthouses will continue to operate under space weather conditions. Again, like buoys and MFAs, lighthouses are becoming prominent locations on which to mount additional services, such as AtoN AIS and differential services.

GLA lighthouses are configured to operate on battery backup should mains power be lost due to geomagnetic storms, sites are configured to operate for several weeks without maintenance. It should be noted that each lighthouse may be supported for different durations, based on the AtoNs fitted at that site.

\section{Aton monitoring systems}

Monitoring is conducted to fulfil each GLAs navigational, operational and legal requirement [3] and is necessary in order to calculate an AtoN's availability. 
Each GLA utilises a number of different communication systems, depending on the location and operational requirement. These include PSTN, ADSL, satellite communications, GSM and point-to-point fixed radio links to provide information from one site to another and from AtoNs to shore.

Each of these may be affected by space weather events, whether directly through changes to the atmosphere or by an increase in radio noise. Further work is required in this area to assess the full impact on each system, but it is anticipated that the effects will be minor and with a low probability.

It should be noted that the GLAs' ability to monitor an AtoN does not affect its performance from the mariner's perspective. All GLA AtoNs are designed to be autonomous so that they can remain operational should communications be lost.

\section{MITIGATION}

Mitigating the effect of space weather is a difficult process. The best form of mitigation is to stop the event from occurring and clearly this is not an option in this case. Therefore the remaining options are to:

— raise awareness of space weather events and their impact;

- provide notice of impending storms;

— minimise or prevent the effect of their impact.

\section{A warenes}

Space Weather has been in the news recently following several significant solar flares during March and the recent agreement between the United States and the United Kingdom to work closely on space weather monitoring [13].

Increasing awareness of the impact on maritime safety is two fold; firstly the GLAs must understand the impact and the potential vulnerabilities to their AtoN provision so that we can do what we can to provide a robust and reliable service; secondly the mariner must be made aware as to the potential impact of space weather, not only on the GLA AtoN provision but also to their own vessel systems.

The first of these tasks is partly addressed by this work and will be further developed over time. The second task will need to be addressed both by the GLAs and other maritime stakeholders within the UK and Ireland. The GLAs are currently working with these stakeholders to identify the best means of raising awareness to ensure any alerts are meaningful and timely. 


\section{Monitoring space weather}

Awareness of impending space weather events would enable mariners and service providers alike to activate alternative systems or to stop critical operations. However, given the speed of some events, this is not always possible.

The GLAs are not in a position to monitor space weather events. There are a number of organisations around the world who undertake this role, one of which is the US Space Weather Prediction Centre (SWPC) operated by the United States National Oceanic and Atmospheric Administration (NOAA).

SWPC personnel monitor the Sun's activity through many different sensors, in an effort to identify storms before they occur and to understand the impact when they do. When an event is identified, the SWPC issue email alerts to warn users. While it's possible to predict and observe whether an event will affect Earth, science is not currently able to predict where on Earth the effects will be felt and therefore warnings are general in nature and in effect, the whole Earth is put on notice.

It is recognised that in order for these alerts to be useful, the GLAs need to understand the strength category that will affect their service provision and identify the correct means for providing information to the mariner so that it remains useful, without providing false alarms.

\section{Minimising and preventing the effects}

Minimising or preventing the effect of space weather can be quite difficult and would need to be considered for each individual system and service.

For radio communications and data transmission it may not be possible to mitigate the effect of a radio blackout as noise is provided across the radio spectrum. However, it is hypothesised that different systems would be affected for different durations and in this case, using alternative frequencies or systems may provide a significant benefit.

When considering geomagnetic storms and their affect on the ionosphere, the use of dual or multiple frequency (D)GNSS receivers should provide a level of mitigation and enable the mariner to minimise some of the effects.

\section{CONCLUSIONS}

Low strength space weather events are fairly constant and generally go by unnoticed, without affecting the mariner or the GLA AtoN service provision. 
Storms with the strength to affect these operations are very infrequent, with the most extreme storms referred to as once in 300 or 500 year events. However, it is important to note that they are stochastic in nature and as such could occur at any time.

Space weather events, could affect GNSS derived position, navigation and timing information by affecting the satellite's operation or reported position, along with affecting the user's ability to receive the transmitted signals. At the most extreme, the receiver's tracking of GNSS signals could be lost due to interference and noise, resulting in similar conditions as to those experienced in the GLA jamming trials. eLoran signals may also be affected by space weather, but with different failure modes expected. Loran services remained operational during the 2006 radio storm.

Of the GLA AtoN service provision, only AtoN AIS units appear to be significantly affected as it is not known how they would respond if GPS were denied. Further work is required in this area and to investigate the provision of integrity information.

Physical AtoNs, such as lighthouses, buoys and MFAs will continue to operate and are resilient to space weather events. GLA AtoNs are fitted with alternative power back up systems, should the national power network become unavailable.

Radionavigation aids are relatively robust; the GLA DGPS network and eLoran services can be affected but will alert the mariner if that is the case. DGPS will help mitigate increased GNSS position errors and warn the mariner if they become too large.

The GLAs ability to monitor the performance of their AtoNs could be affected, however this becomes an internal operational concern and would not affect the AtoNs ability to perform.

The information in this paper should be kept in context. Significant space weather events are very rare and the world at large is still learning about them. A recent UK Government [15] report rightly notes (...) space weather science is a relatively young field and its impacts on modern society are only recently coming to the fore as our dependence on technologies vulnerable to solar phenomena increases. Therefore significant work is continuing to better understand and plan in a proportionate way - for the expected impacts of a severe space weather event. As space weather is a young field, the findings in this report may change as knowledge develops. The GLAs will continue to learn about space weather and its affects on their AtoN service to ensure they continue to keep all mariners safe within their waters. 


\section{REFERENCES}

[1] American Meteorological Society, Satellite Navigation \& Space Weather: Understanding the Vulnerability \& Building Resilience, Policy workshop report, March 2011.

[2] Cerruti et al, Effect of intense December 2006 solar radio burst on GPS receivers, Space Weather Journal, 2008, Vol. 6.

[3] GLA Joint Radionavigation Requirements Policies, September 2008.

[4] Grant A., Williams P., Ward N., Basker S., GPS Jamming and the impact on maritime navigation, Journal of the Royal Institute of Navigation, 62, April 2009.

[5] ICAO, Space Weather effects in regards to international air navigation.

[6] Jakowski N. et al, Space Weather Effects in the Ionosphere and their Impact on Positioning, ESA workshop paper.

[7] Kintner P., A beginner's guide to Space Weather and GPS, 21/2/2008.

[8] Murphy T., Boeing, Space weather effects on aviation - an overview, ION GNSS 2011, Portland 2011.

[9] NOAA Space Weather Scales, http://www.swpc.noaa.gov/NOAAscales.

[10] NOAA Technical Memorandum, Halloween Space Weather storms of 2003, OAR SEC-88, June 2004.

[11] Shaw G., Ionospheric Effects on GNSS (Phase 1), GLA R\&RNAV Report, RPT-08-GS-10.

[12] Thompson A. P. et al, Surface Electric Fields and Geomagnetically Induced Currents in the Scottish Power Grid During the $30^{\text {th }}$ October 2003 Geomagnetic Storm, British Geological Survey.

[13] UK \& US Collaboration on space weather monitoring, http://www.number10.gov.uk/ news/uk-us-higher-education-science-and-innovation-collaboration/.

[14] UK Cabinet Office, National Risk register of Civil Emergencies, 2012 edition.

[15] UK House of Commons Defence Committee, Developing Threats: Electro-Magnetic Pulses (EMP), Tenth report of session 2010-12.

[16] United States National Space Weather Program Implementation Plan, $2^{\text {nd }}$ Edition, July 2000.

[17] US National Space Weather Program: The implementation plan, $2^{\text {nd }}$ ed. Committee for Space Weather, Office of the Federal Coordinator for Meteorological Services and Supporting Research Rep.OFCM-P31-2000, available online at www.ofcm.gov/nswp-ip/pdf/nswpip.pdf. 
[18] US Space Weather Prediction Centre, http://www.swpc.noaa.gov/aurora/ globeNE.html.

[19] http://www.sott.net/articles/show/242895-A-Perfect-Solar-Superstorm-The-1859Carrington-Event.

Received April 2012

Reviewed October 2012 